\title{
LINEAR MAPS PRESERVING THE ISOMORPHISM CLASS OF LATTICES OF INVARIANT SUBSPACES
}

\author{
ALI A. JAFARIAN, LEIBA RODMAN, AND PETER ŠEMRL
}

(Communicated by David R. Larson)

\begin{abstract}
Let $\mathcal{V}$ be an $n$-dimensional complex linear space and $\mathcal{L}(\mathcal{V})$ the algebra of all linear transformations on $\mathcal{V}$. We prove that every linear map on $\mathcal{L}(\mathcal{V})$, which maps every operator into an operator with isomorphic lattice of invariant subspaces, is an inner automorphism or an inner antiautomorphism multiplied by a nonzero constant and additively perturbed by a scalar type operator. The same result holds if we replace the lattice of invariant subspaces by the lattice of hyperinvariant subspaces or the set of reducing subspaces. Some of these results are extended to linear transformations of finite-dimensional linear spaces over fields other than the complex numbers. We also characterize linear bijective maps on the algebra of linear bounded operators on an infinite-dimensional complex Hilbert space which have similar properties with respect to the lattice of all invariant subpaces (not necessarily closed).
\end{abstract}

\section{INTRODUCTION}

The subject of linear preservers, that is, linear maps on spaces of matrices and operators which preserve certain properties, has been extensively studied in the last few decades. An interested reader is referred to the partial list of references, containing more than 200 titles, in Linear and Multilinear Algebra, volume 33, (1992), pp.121-129. In particular, Jafarian and Sourour [JaS] characterized linear maps preserving any of the following functions: the lattice of invariant subspaces, the lattice of hyperinvariant subspaces, the set of reducing subspaces. In this paper we propose the more general and more difficult problem of characterizing linear maps preserving these partially ordered sets only up to an isomorphism.

Let us first fix notation and terminology. The algebra of all linear transformations on a vector space $\mathcal{V}$ over a field $F$ is denoted by $\mathcal{L}(\mathcal{V})$. If $T \in \mathcal{L}(\mathcal{V})$, and if $\mathcal{W}$ is a linear subspace in $\mathcal{V}$, we call $\mathcal{W}$ invariant (respectively hyperinvariant, respectively reducing) for $T$ if $T \mathcal{W} \subset \mathcal{W}$ (respectively $A \mathcal{W} \subset \mathcal{W}$ for every $A$ commuting with $T$, respectively $T \mathcal{W} \subset \mathcal{W}$ and $T \mathcal{U} \subset \mathcal{U}$ for some linear subspace $\mathcal{U} \subset \mathcal{V}$ satisfying $\mathcal{V}=\mathcal{W} \oplus \mathcal{U}$; here and elsewhere in the paper we use the notation $\oplus$ to designate a direct sum in the algebraic sense). The set of all such linear subspaces is denoted by lat $T$ (respectively hyperlat $T$, respectively $\operatorname{red} T$ ). We write

Received by the editors April 21, 1997.

1991 Mathematics Subject Classification. Primary 47A15.

The first author was supported by a grant from the University of New Haven.

The second author was partially supported by NSF Grant DMS-9500924.

The third author was supported by a grant from the Ministry of Science of Slovenia. 
lat $T \approx$ lat $S$ (hyperlat $T \approx$ hyperlat $S$ ) if there exists a lattice isomorphism of lat $T$ onto lat $S$ (hyperlat $T$ onto hyperlat $S$ ). Note that in general $\operatorname{red} T$ is not a lattice. In this case we write $\operatorname{red} T \approx \operatorname{red} S$ if there exists a bijective map $\tau: \operatorname{red} T \rightarrow \operatorname{red} S$ such that for every $\mathcal{U}, \mathcal{W} \in \operatorname{red} T$ we have $\tau(\mathcal{U}) \subset \tau(\mathcal{W})$ if and only if $\mathcal{U} \subset \mathcal{W}$.

\section{Finite-Dimensional CASE}

In this section we will formulate and prove the results on linear maps preserving lattices of invariant subspaces up to an isomorphism in the finite-dimensional case. We decided to deal with this case separately because of several reasons. First, the results in the finite-dimensional case are essentially different from the infinitedimensional case. Some of the proofs of the infinite-dimensional results do not work in the finite-dimensional case unless we impose some restrictions such as the assumption that the dimension of the underlying linear space is at least five. And finally, much shorter proofs are available in this special case.

We state our main results in the finite-dimensional case for the complex vector spaces first.

Theorem 2.1. Let $\mathcal{V}$ be an $n$-dimensional complex vector space and $\varphi: \mathcal{L}(\mathcal{V}) \rightarrow$ $\mathcal{L}(\mathcal{V})$ a linear map. Then the following statements are equivalent:

(a) lat $T \approx \operatorname{lat} \varphi(T)$ for every $T \in \mathcal{L}(\mathcal{V})$,

(b) hyperlat $T \approx$ hyperlat $\varphi(T)$ for every $T \in \mathcal{L}(\mathcal{V})$,

(c) $\operatorname{red} T \approx \operatorname{red} \varphi(T)$ for every $T \in \mathcal{L}(\mathcal{V})$,

(d) there exist a nonzero complex number $\alpha$, an invertible $S \in \mathcal{L}(\mathcal{V})$, and a linear functional $f$ on $\mathcal{L}(\mathcal{V})$ such that either

$$
\varphi(T)=\alpha S T S^{-1}+f(T) I, \quad T \in \mathcal{L}(\mathcal{V}),
$$

or

$$
\varphi(T)=\alpha S T^{t} S^{-1}+f(T) I, \quad T \in \mathcal{L}(\mathcal{V}),
$$

where $T^{t}$ is the transpose of $T$ with respect to a fixed basis,

(e) the map $\varphi$ preserves the Jordan structure, i.e., for every $T \in \mathcal{L}(\mathcal{V})$ the operators $T$ and $\varphi(T)$ have the same Jordan structure.

In connection with (e), two linear operators $S$ and $T$ on $\mathcal{V}$ are said to have the same Jordan structure if the number of distinct eigenvalues of $S$ and $T$ is the same and there exists a bijection $\beta: \sigma(S) \rightarrow \sigma(T)$ such that the algebraic multiplicities of $\lambda$ as an eigenvalue of $S$ coincide with the algebraic multiplicities of $\beta(\lambda)$ as an eigenvalue of $T$, for every $\lambda \in \sigma(S)$. This notion was studied in [GLR], [GR], [O].

Proof. First of all observe that the equivalence of (a) and (e) is given in Theorem 16.1.2 of [GLR]. Obviously, (d) implies each of (a), (b), and (c). We will prove the reverse implications simultaneously. Defining

$$
\psi(T)=\varphi(T)-(1 / n)(\operatorname{tr} \varphi(T)-\operatorname{tr} T) I
$$

we get a linear map $\psi$ satisfying $\operatorname{tr} \psi(T)=\operatorname{tr} T$ and $\psi(I)=I$. Clearly, $\psi$ satisfies one of the properties (a), (b), or (c) if and only if $\varphi$ does.

Next, we will prove that $\psi$ is invertible. Assume first that $\psi$ satisfies (a). It is easy to see that lat $T$ is isomorphic to the lattice of all subspaces of $\mathcal{V}$ if and only if $T=\beta I$ for some complex number $\beta$. Hence, $\psi(T)=0$ implies that $T=\beta I$ for some $\beta$. But $\psi$ preserves the trace, and therefore, $\beta=0$. If $\psi$ satisfies (b) or (c) we 
get the bijectivity using a similar argument and the fact that $T=\beta I$ if and only if hyperlat $T=\{\{0\}, \mathcal{V}\}$ if and only if $\operatorname{red} T$ is the lattice of all subspaces of $\mathcal{V}$.

Observe that if $N \in \mathcal{L}(\mathcal{V})$ is a nilpotent of nilindex $n$, then lat $N$ (hyperlat $N$ ) is a chain and $\operatorname{red} N=\{\{0\}, \mathcal{V}\}$. It is easy to see that if lat $T$ (hyperlat $T$ ) is a chain or if $\operatorname{red} T=\{\{0\}, \mathcal{V}\}$, then $T=\beta I+M$ with $M$ being nilpotent. Applying once again the fact that $\psi$ preserves the trace, we conclude that $\psi$ maps the set of all nilpotent operators of nilindex $n$ into itself. The set of all nilpotents of nilindex $n$ is dense in the closed subset $\mathcal{N} \subset \mathcal{L}(\mathcal{V})$ consisting of all nilpotents from $\mathcal{L}(\mathcal{V})$. Consequently, $\psi$ maps nilpotents into nilpotents. Since the set $\mathcal{S} \mathcal{L}(\mathcal{V})$ of all linear transformations on $\mathcal{V}$ with zero trace is the linear span of $\mathcal{N}, \psi$ maps $\mathcal{S L}(\mathcal{V})$ into itself. Now applying the structural result for such maps [BPW] (for a short elementary proof of this result see [Sem]) we see that there exist an invertible $S \in \mathcal{L}(\mathcal{V})$ and a nonzero $\alpha \in \mathbb{C}$ such that either

$$
\psi(T)=\alpha S T S^{-1}, \quad T \in \mathcal{S L}(\mathcal{V}),
$$

or

$$
\psi(T)=\alpha S T^{t} S^{-1}, \quad T \in \mathcal{S} \mathcal{L}(\mathcal{V}),
$$

where $T^{t}$ is the transpose of $T$ with respect to a fixed basis. Define $\theta$ by $\theta(T)=$ $\psi(T)+((\alpha-1) / n) \operatorname{tr}(T) I$. Then, $\theta(T)=\psi(T)$ for every $T \in \mathcal{S} \mathcal{L}(\mathcal{V})$, and $\theta(I)=\alpha I$. Since $I$ and $\mathcal{S} \mathcal{L}(\mathcal{V})$ span $\mathcal{L}(\mathcal{V})$, we have either

$$
\theta(T)=\alpha S T S^{-1}, \quad T \in \mathcal{L}(\mathcal{V})
$$

or

$$
\theta(T)=\alpha S T^{t} S^{-1}, \quad T \in \mathcal{L}(\mathcal{V})
$$

This completes the proof.

Essentially the same proof shows that Theorem 2.1 is valid for $n$-dimensional vector spaces over any algebraically closed field provided the characteristic of the field does not divide $n$ (this provision is needed to allow us the reduction to the case when $\psi$ preserves traces). The denseness argument used in the proof above to assert that $\psi$ maps nilpotents to nilpotents can be replaced by another argument that does not use the topology of complex numbers (see Proposition 2.6 below).

In the rest of this section we will show that a part of Theorem 2.1 concerning the equivalence of (a) and (d) remains valid also for many fields that are not algebraically closed. We develop some preliminary results first. Throughout the rest of this section, $\mathcal{V}$ is a finite-dimensional vector space over a field $F$ having dimension $n$.

Proposition 2.2. Let $T$ be a linear transformation on $\mathcal{V}$. Then every one-dimensional subspace of $\mathcal{V}$ is $T$-invariant if and only if $T=\beta I$ for some $\beta \in F$.

The proof is an easy exercise.

Proposition 2.3. If $F$ is infinite, and if $\mathcal{V}=Z_{1} \cup \cdots \cup Z_{p}$ is a union of a finite number of subspaces $Z_{1}, \cdots, Z_{p}$, then in fact $\mathcal{V}=Z_{j}$ for some $Z_{j}$.

Proof. This proposition is probably well-known. We indicate a proof anyway. Arguing by contradiction, we can assume that $\operatorname{dim}\left(Z_{j}\right)=n-1$ for $j=1, \cdots, p$. We 
also assume that $\mathcal{V}=F^{n}$ for some $n$, the vector space of $n$-component row vectors. Then

$$
Z_{j}=\left\{x=\left[x_{1}, x_{2}, \cdots, x_{n}\right] \in F^{n}: \sum_{k=1}^{n} x_{k} \alpha_{k j}=0\right\}
$$

for some $\alpha_{k j} \in F$, where at least one of $\alpha_{1 j}, \alpha_{2 j}, \cdots, \alpha_{n j}$ is different from zero. We have to find a $y=\left[y_{1}, y_{2}, \cdots, y_{n}\right] \in F^{n}$ such that all entries of $y A$ are nonzero, where $A=\left[\alpha_{k j}\right]$ is an $n \times p$ matrix. For this purpose we can assume without loss of generality that $A$ is reduced to the row reduced echelon form, in which case the construction of $y$ proceeds starting with $y_{1}=1$, and if $y_{1}, \cdots, y_{q-1}$ are already determined, the selection of $y_{q} \neq 0$ is arbitrary subject to a finite number of inequalities of the form $\sum_{k=1}^{q} \gamma_{k} y_{k} \neq 0$, where $\gamma_{k} \in F$ and $\gamma_{q} \neq 0$. Since $F$ is infinite, this finite number of inequalities can always be satisfied.

Proposition 2.4. A linear transformation $T$ on $\mathcal{V}$ has a Jordan form (i.e., there is a basis in $\mathcal{V}$ with respect to which $T$ is given by a matrix in a Jordan normal form) if and only if lat $T$ contains a chain of $n+1$ elements.

Proof. If $T$ has a Jordan form, then obviously lat $T$ contains a chain of $n+1$ elements. Conversely, let

$$
\{0\}=\mathcal{V}_{0} \subset \mathcal{V}_{1} \subset \cdots \subset \mathcal{V}_{n-1} \subset \mathcal{V}_{n}=\mathcal{V}
$$

be a chain of $n+1$ elements in lat $T$. Selecting $x_{k} \in \mathcal{V}_{k} \backslash \mathcal{V}_{k-1}$ we obtain a basis in $\mathcal{V}$ with respect to which $T$ has an upper triangular form. In particular, all eigenvalues of $T$ belong to $F$. Now a standard proof of the Jordan normal form shows that $T$ has a Jordan form.

Proposition 2.5. If lat $T$ is isomorphic (as a lattice) to the lattice of all subspaces in $\mathcal{V}$, then $T$ is a scalar multiple of $I$.

Proof. We assume that $F$ is infinite (otherwise, the lattice of all subspaces in $\mathcal{V}$ is finite and therefore cannot be isomorphic to its proper sublattice, and we are done by Proposition 2.2). Since lat $T$ contains a chain of $n+1$ elements, by Proposition $2.4 T$ has a Jordan form, and without loss of generality we can assume that $T$ is given by a Jordan matrix.

Since the lattice of lat $(\mathcal{V})$ of all subspaces of $\mathcal{V}$ has the property that for every $X \in \operatorname{lat}(\mathcal{V})$ there is $Y \in \operatorname{lat}(\mathcal{V})$ such that $X+Y=\mathcal{V}, X \cap Y=\{0\}$, the lattice lat $T$ has the same property, i.e., every $T$-invariant subspace is reducing. Using the Jordan form of $T$, it is easy to see that $T$ must be a diagonal matrix. Thus, lat $T$ is isomorphic to a direct $\operatorname{sum} \operatorname{lat}\left(\mathcal{V}_{1}\right) \oplus \cdots \oplus \operatorname{lat}(\mathcal{V})_{p}$, where $\mathcal{V}_{1}, \cdots, \mathcal{V}_{p}$ are linearly independent subspaces of $\mathcal{V}$ such that $\mathcal{V}_{1}+\cdots+\mathcal{V}_{p}=\mathcal{V}$. Let

$$
\varphi: \operatorname{lat} T \rightarrow \operatorname{lat}\left(\mathcal{V}_{1}\right) \oplus \cdots \oplus \operatorname{lat}\left(\mathcal{V}_{p}\right)
$$

be an isomorphism. Let lat $\left.\mathcal{V}_{j}\right)$ be the set of one-dimensional subspaces in $\mathcal{V}_{j}$, and let

$$
Q_{j}=\varphi^{-1}\left(\{0\} \oplus \cdots \oplus\{0\} \oplus \operatorname{lat}_{1}\left(\mathcal{V}_{j}\right) \oplus \cdots \oplus\{0\}\right) .
$$


Then $Q_{1}, \cdots, Q_{p}$ form a disjoint partition of $\operatorname{lat}_{1}(\mathcal{V})$. Since $\varphi$ is a lattice isomorphism, it follows that for every pair of linearly independent vectors $x, y$ such that $\operatorname{Span}\{x\}, \operatorname{Span}\{y\} \in Q_{1}$, the two-dimensional subspace $\operatorname{Span}\{x, y\}$ does not contain any element of the set $Q_{2} \cup \cdots \cup Q_{p}$. It follows that the set

$$
Z_{1}=\left\{x: \operatorname{Span}\{x\} \in Q_{1}\right\} \cup\{0\}
$$

is actually a subspace of $\mathcal{V}$. Analogously, all the sets $Z_{j}, j=2, \cdots, p$ (which are defined analogously to $Z_{1}$, replacing the subscript 1 by the subscript $j$ ), are subspaces of $\mathcal{V}$. Clearly,

$$
\mathcal{V}=Z_{1} \cup Z_{2} \cup \cdots \cup Z_{p}
$$

By Proposition 2.3, $p=1$, i.e., $T$ is a scalar multiple of $I$.

Proposition 2.6. Let $S$ and $T$ be two linear transformations on $\mathcal{V}$ having Jordan forms. Assume that either $F$ is infinite or $F$ is finite but has at least $n$ elements. If $T$ has only one distinct eigenvalue, and $S$ has at least two distinct eigenvalues, then lat $T$ and lat $S$ are not isomorphic.

Proof. Without loss of generality we assume that $T$ is nilpotent. Argue by contradiction, and assume that $S$ has $p>1$ distinct eigenvalues, but the lattices lat $T$ and lat $S$ are isomorphic. Consider first the case when $F$ is infinite. Then we argue as in the proof of Proposition 2.5, $\operatorname{replacing} \operatorname{lat}_{1}(\mathcal{V})$ by $\operatorname{lat}_{1}(\operatorname{Ker} T)$ and replacing each $\operatorname{lat}_{1}\left(\mathcal{V}_{j}\right)$ by $\operatorname{lat}_{1}\left(\operatorname{Ker}\left(S-\lambda_{j} I\right)\right)$, where $\lambda_{1}, \cdots, \lambda_{p}$ are all the distinct eigenvalues of $S$.

Assume now that $F$ has exactly $\alpha$ elements, where $n \leq \alpha<\infty$. Use the easily verifiable fact that a $q$-dimensional subspace over $F$ has exactly $\left(\alpha^{q}-1\right) /(\alpha-1)$ one-dimensional subspaces. Now if we had that lat $T \approx \operatorname{lat} S$, then in view of the fact just mentioned we would have the equality

$$
\left(\alpha^{q}-1\right) /(\alpha-1)=\left(\alpha^{q_{1}}-1\right) /(\alpha-1)+\cdots+\left(\alpha^{q_{p}}-1\right) /(\alpha-1),
$$

where $q=\operatorname{dim} \operatorname{Ker} T$ and $q_{j}=\operatorname{dim} \operatorname{Ker}\left(S-\lambda_{j}\right.$ ) (as before, $\lambda_{1}, \cdots, \lambda_{p}$ are the distinct eigenvalues of $S$ ). Since $p>1$ by assumption, we have $q_{j}<q$ for all $j$. Also, obviously, $p \leq n$. Now

$$
\begin{aligned}
\sum_{j=1}^{p}\left(\alpha^{q_{j}}-1\right) & \leq \sum_{j=1}^{p}\left(\alpha^{q-1}-1\right)=p\left(\alpha^{q-1}-1\right) \\
& <p \alpha^{q-1}-1 \leq n \alpha^{q-1}-1 \leq \alpha^{q}-1,
\end{aligned}
$$

a contradiction with (2.1).

Theorem 2.7. Let $\mathcal{V}$ be an $n$-dimensional vector space over a field $F$, and let $\varphi: \mathcal{L}(\mathcal{V}) \rightarrow \mathcal{L}(\mathcal{V})$ be a linear map. Assume that $F$ has at least $n$ elements and that the characteristic of $F$ does not divide $n$. Then lat $T \approx \operatorname{lat} \varphi(T)$ for every $T \in \mathcal{L}(\mathcal{V})$ if and only if the condition (d) of Theorem 2.1 is satisfied.

The proof follows the pattern of the proof of Theorem 2.1. Use Proposition 2.5 to prove that if $\varphi$ satisfies lat $T \approx \operatorname{lat} \varphi(T)$ for every $T \in \mathcal{L}(\mathcal{V})$, then $\varphi$ is bijective, and use Proposition 2.6 to prove that such $\varphi$ maps nilpotents into nilpotents. The result of $[\mathrm{BPW}]$ is applicable for any field having at least $n$ elements. 


\section{INFINITE-DIMENSIONAL CASE}

Our first result in this section deals with linear maps preserving lattices of invariant subspaces up to an isomorphism. Note that in this section lat $T$ denotes the lattice of all (not necessarily closed) subspaces of $T$.

Theorem 3.1. Let $H$ be an infinite-dimensional complex separable Hilbert space and $\mathcal{B}(H)$ the algebra of all bounded linear operators on $H$. Assume that $\varphi$ : $\mathcal{B}(H) \rightarrow \mathcal{B}(H)$ is a bijective linear map such that lat $T \approx$ lat $\varphi(T)$ for every $T \in \mathcal{B}(H)$. Then there exist a nonzero complex number $\alpha$, an invertible $S \in \mathcal{B}(H)$, and a linear functional $f$ on $\mathcal{B}(H)$ such that

$$
\varphi(T)=\alpha S T S^{-1}+f(T) I
$$

for every $T \in \mathcal{B}(H)$.

Remarks. In the finite-dimensional case a linear transformation that maps every $T$ into an operator with isomorphic lattice of invariant subspaces is either an automorphism multiplied by a nonzero scalar and perturbed by a scalar-type operator, or an antiautomorphism multiplied by a nonzero scalar and perturbed by a scalartype operator. In the infinite-dimensional case the second possibility cannot occur. In this case we need the additional assumption that $\varphi$ is bijective. Note that we do not assume continuity of $\varphi$, and consequently, we get a functional $f$ which need not be continuous either. However, $S$ is continuous and $\varphi$ can get some discontinuity only through the functional $f$. It would also be natural to consider linear maps that preserve up to an isomorphism the lattice of all closed invariant subspaces. We believe that this problem is much more difficult because we do not know the answer to the invariant subspace problem. We note, however, that if the invariant subspace problem has the affirmative solution, i.e., every linear bounded operator on a complex Hilbert space of dimension at least 2 has a nontrivial invariant subspace, then the result of Theorem 3.1 is valid also for the lattice of closed invariant subspaces (with essentially the same proof).

For the proof of the above result we shall need some lemmas. We denote by lat $_{n}$ and by lat $\infty$ the lattices of all linear (not necessarily closed) subspaces of $n$ dimensional Hilbert space $E_{n}$ and infinite-dimensional separable Hilbert space $E$, respectively.

Lemma 3.2. Let $\mathcal{V}$ be a complex vector space, and let $T \in \mathcal{L}(\mathcal{V})$ be an operator satisfying lat $T \approx \operatorname{lat}_{n}$ (lat $T \approx$ lat $_{\infty}$ ). Then $T$ is a scalar multiple of the identity operator and $\operatorname{dim} \mathcal{V}=n(\operatorname{dim} \mathcal{V}=\infty)$.

Proof. Assume first that there exists $x \in \mathcal{V}$ such that $x, T x, T^{2} x, \ldots$ are linearly independent. Then $\operatorname{Span}\left\{x, T x, T^{2} x, \ldots\right\} \subset \mathcal{V}$ is an invariant subspace for $T$. Every nonzero element of lat ${ }_{n}\left(\right.$ lat $\left._{\infty}\right)$ contains at least one minimal nonzero element. On the other hand, it is easy to see that $\operatorname{Span}\left\{x, T x, T^{2} x, \ldots\right\}$ contains no nonzero minimal $T$-invariant subspace. This contradiction shows that every $x \in \mathcal{V}$ is contained in a minimal finite-dimensional $T$-invariant subspace, say $\mathcal{V}_{x} \subset \mathcal{V}$. Let $\tau:$ lat $T \rightarrow$ lat $_{n}$ (lat ${ }_{\infty}$ ) be a lattice isomorphism. Denote $\mathcal{W}=\tau\left(\mathcal{V}_{x}\right) \subset E_{n}(E)$. Then $\operatorname{lat}\left(T_{\mid \mathcal{V}_{x}}\right) \approx \operatorname{lat} \mathcal{W}$. If $\operatorname{dim} \mathcal{V}_{x}=p$, then $\operatorname{lat}\left(T_{\mid \mathcal{V}_{x}}\right)$ contains a chain with $p+1$ elements and no chains with more than $p+1$ elements. It follows that $\operatorname{dim} \mathcal{W}=p$. Applying Proposition 2.5 (of course, a shorter proof of this proposition is available in the complex case) we see that $T_{\mid \mathcal{V}_{x}}$ is a scalar multiple of the identity operator on 
$\mathcal{V}_{x}$. As $x \in \mathcal{V}$ was an arbitrary vector, we conclude that $T$ is a scalar multiple of the identity operator. Comparing the cardinality of the chains with the maximal length in lat $T$ and $\operatorname{lat}_{n}\left(\operatorname{lat}_{\infty}\right)$ we finally conclude that $\operatorname{dim} \mathcal{V}=n(\operatorname{dim} \mathcal{V}=\infty)$

Lemma 3.3. Let $A \in \mathcal{B}(H)$. Then the following are equivalent:

(a) $A=\alpha P+\beta I$ with $\alpha \neq 0, P^{2}=P$, and $\operatorname{rank} P=n$,

(b) lat $A \approx \operatorname{lat}_{n} \oplus \operatorname{lat}_{\infty}$.

Proof. Assume first that (a) is satisfied. Then $K \in$ lat $A$ implies that $K \in$ lat $P$. Hence, $P K \subset K,(I-P) K \subset K$, and $K=P K \oplus(I-P) K$. From here one can easily see that (b) holds true.

To prove the converse we denote by $\tau$ isomorphism of lat $A$ onto lat ${ }_{n} \oplus \operatorname{lat}_{\infty}$. Let $K_{1}=\tau^{-1}\left(E_{n} \oplus\{0\}\right)$ and $K_{2}=\tau^{-1}(\{0\} \oplus E)$. Then $K_{1}$ and $K_{2}$ are (not necessarily closed) $A$-invariant subspaces of $H$ such that $H=K_{1} \oplus K_{2}$. Applying Lemma 3.2 we see that $\operatorname{dim} K_{1}=n$ and that the restriction of $A$ to $K_{1}$ is a scalar multiple of the identity, say $\delta I_{K_{1}}$. Similarly, $A_{\mid K_{2}}=\beta I_{K_{2}}$ with $\beta \neq \delta$. Clearly, $K_{2}=\operatorname{Ker}(A-\beta I)$, and consequently, $K_{2}$ is closed. Set $\alpha=\delta-\beta$ to conclude the proof.

In almost the same way one can prove the following lemma.

Lemma 3.4. Let $A \in \mathcal{B}(H)$. Then the following are equivalent:

(a) $A=\alpha P+\beta I$ with $\alpha \neq 0, P^{2}=P$, and $\operatorname{dim} \operatorname{Im} P=\operatorname{dim} \operatorname{Ker} P=\infty$,

(b) lat $A \approx \operatorname{lat}_{\infty} \oplus \operatorname{lat}_{\infty}$.

Lemma 3.5. Let $Q_{1}, Q_{2}, Q \in \mathcal{B}(H)$ be idempotents with $\operatorname{rank} Q_{1}=\operatorname{rank} Q_{2}=1$ and $\operatorname{rank} Q=2$. Assume that $\alpha, \alpha_{1}, \alpha_{2}$ are nonzero complex numbers such that $\alpha Q=\alpha_{1} Q_{1}+\alpha_{2} Q_{2}$. Then $\alpha=\alpha_{1}=\alpha_{2}$ and $Q_{1} Q_{2}=Q_{2} Q_{1}=0$.

Proof. We have $Q=\beta_{1} Q_{1}+\beta_{2} Q_{2}$ with $\beta_{i}=\left(\alpha_{i} / \alpha\right), i=1,2$. Using trace we see that $\beta_{1}+\beta_{2}=2$. The equation $Q^{2}=Q$ can be rewritten as

$$
\left(\beta_{1}^{2}-\beta_{1}\right) Q_{1}+\left(\beta_{2}^{2}-\beta_{2}\right) Q_{2}+\beta_{1} \beta_{2}\left(Q_{1} Q_{2}+Q_{2} Q_{1}\right)=0 .
$$

Multiplying this equation by $Q_{1}$ from the left, then multiplying it once again by $Q_{1}$, this time from the right, and comparing the obtained relations we arrive at $\left(\beta_{2}^{2}-\beta_{2}+\beta_{1} \beta_{2}\right)\left(Q_{1} Q_{2}-Q_{2} Q_{1}\right)=0$. Using $\beta_{1}=2-\beta_{2}$ and $\beta_{2} \neq 0$ we get that $Q_{1}$ and $Q_{2}$ commute. They are rank one operators and using their matrix representations we conclude that either $Q_{1}=Q_{2}$ or $Q_{1} Q_{2}=Q_{2} Q_{1}=0$. The first possibility is in a contradiction with $\operatorname{rank} Q=2$. So, $Q_{1}$ and $Q_{2}$ are perpendicular, and consequently, $\beta_{1}^{2} Q_{1}+\beta_{2}^{2} Q_{2}=\beta_{1} Q_{1}+\beta_{2} Q_{2}$. Multiplying by $Q_{1}$ we obtain $\beta_{1}^{2}=\beta_{1}$ which yields that $\beta_{1}=1$. Similarly we get $\beta_{2}=1$. This completes the proof.

By $\mathcal{F}(H) \subset \mathcal{B}(H)$ we denote the ideal of all finite rank operators. We will also need Lemma 1 from $[\mathrm{BrS}]$ which states that if $\theta: \mathcal{F}(H) \rightarrow \mathcal{F}(H)$ is a linear map which maps every idempotent into an idempotent, then $\theta$ is a Jordan homomorphism, that is, $\theta\left(A^{2}\right)=(\theta(A))^{2}$ for every $A \in \mathcal{F}(H)$.

Proof of Theorem 3.1. By Lemma 3.3 every idempotent $P$ of rank one is sent by $\varphi$ into an operator of the form $\alpha Q+\beta I$ with $\alpha \neq 0, \operatorname{rank} Q=1$, and $Q^{2}=Q$. Let us show that $\alpha$ is independent of $P$. First we will consider the case that $P_{1}$ and $P_{2}$ are perpendicular idempotents of rank one. Denote $\varphi\left(P_{1}\right)=\alpha_{1} Q_{1}+\beta_{1} I$ and $\varphi\left(P_{2}\right)=\alpha_{2} Q_{2}+\beta_{2} I$. As $P=P_{1}+P_{2}$ is an idempotent of rank two, we have by 
Lemma 3.3 that $\varphi(P)=\alpha Q+\beta I$ for some nonzero complex number $\alpha$ and some idempotent $Q$ of rank two. Hence,

$$
\alpha Q-\alpha_{1} Q_{1}-\alpha_{2} Q_{2}=\left(\beta_{1}+\beta_{2}-\beta\right) I .
$$

The operator on the left-hand side is of finite rank. It follows that $\beta=\beta_{1}+\beta_{2}$. By Lemma 3.5 we get $\alpha_{1}=\alpha_{2}$. Moreover, $Q_{1}$ and $Q_{2}$ are perpendicular. If we have now two arbitrary idempotents $P_{1}$ and $P_{2}$ of rank one with $\varphi\left(P_{1}\right)=\alpha_{1} Q_{1}+\beta_{1} I$ and $\varphi\left(P_{2}\right)=\alpha_{2} Q_{2}+\beta_{2} I$, then we can use an idempotent $P_{3}$ of rank one that is perpendicular to $P_{1}$ as well as to $P_{2}$ to show that $\alpha_{1}=\alpha_{2}$. Multiplying $\varphi$ by an appropriate constant we can assume that $\varphi(P)=Q+\beta I$ for every idempotent $P$ of rank one. Here, of course, $Q$ is also an idempotent of rank one. Let $A \in \mathcal{F}(H)$. Then $A=\sum_{i=1}^{k} \alpha_{i} P_{i}$ for some idempotents of rank one. Hence,

$$
\varphi(A)=B+\beta I
$$

with $B \in \mathcal{F}(H)$. Note that $B$ and $\beta$ are uniquely determined by $A$ since otherwise we would also have $\varphi(A)=B_{1}+\beta_{1} I$ implying that $0 \neq B-B_{1}=\left(\beta_{1}-\beta\right) I$, which is a contradiction because $B-B_{1} \in \mathcal{F}(H)$. Define $f: \mathcal{F}(H) \rightarrow \mathbb{C}$ by $f(A)=\beta$. Here, $\beta$ is defined by (3.1). If $A_{1}, A_{2} \in \mathcal{F}(H)$ and $\alpha_{1}, \alpha_{2} \in \mathbb{C}$, then there exist uniquely determined $B_{1}, B_{2}, B \in \mathcal{F}(H)$ such that $\varphi\left(A_{1}\right)=B_{1}+f\left(A_{1}\right) I, \varphi\left(A_{2}\right)=$ $B_{2}+f\left(A_{2}\right) I$, and $\varphi\left(\alpha_{1} A_{1}+\alpha_{2} A_{2}\right)=B+f\left(\alpha_{1} A_{1}+\alpha_{2} A_{2}\right) I$. Hence, $B-\alpha_{1} B_{1}-$ $\alpha_{2} B_{2}=\left(\alpha_{1} f\left(A_{1}\right)+\alpha_{2} f\left(A_{2}\right)-f\left(\alpha_{1} A_{1}+\alpha_{2} A_{2}\right)\right) I \in \mathcal{F}(H)$. Consequently, $f$ is linear. Clearly, we have $\varphi(I)=\delta I$ for some $\delta \neq 0$. Define $f(I)=0$ and extend $f$ as a linear functional (not necessarily bounded) to $\mathcal{B}(H)$. Replacing $\varphi$ by $\theta(A)=$ $\varphi(A)-f(A) I$ we get $\theta(\mathcal{F}(H)) \subset \mathcal{F}(H)$ and $\theta(I)=\delta I$. Moreover, the restriction of $\theta$ to $\mathcal{F}(H)$ preserves idempotents of rank one. If two such idempotents are perpendicular, then so are their images. Thus, the restriction of $\theta$ to $\mathcal{F}(H)$ preserves idempotents. Applying [BrS, Lemma 1] we conclude that this restriction is a Jordan homomorphism.

Next, we will prove that $\theta: \mathcal{B}(H) \rightarrow \mathcal{B}(H)$ is bijective. If $\theta(A)=0$, then $\varphi(A)=f(A) I$. This yields that $A$ is a scalar multiple of the identity, and so, $f(A)=0$, or equivalently, $\varphi(A)=0$. The injectivity of $\varphi$ yields that $A=0$. To prove the surjectivity we take any $A \in \mathcal{B}(H)$. We know that there exists $B \in \mathcal{B}(H)$ such that $\varphi(B)=A$. It is then clear that $\theta(B+(f(B) / \delta) I)=A$.

It follows that the restriction of $\theta$ to $\mathcal{F}(H)$ considered as a map from $\mathcal{F}(H)$ into itself is bijective. Indeed, all we have to do is to prove that it is surjective. So, take any $A \in \mathcal{F}(H)$. We know that there is $B \in \mathcal{B}(H)$ such that $\theta(B)=A$. As $\theta^{-1}: \mathcal{B}(H) \rightarrow \mathcal{B}(H)$ is a bijective linear map that maps every operator into an operator with isomorphic lattice of all invariant subspaces, we can use the same argument as above to conclude that $B=C+\gamma I$ with $C \in \mathcal{F}(H)$. It follows that $A=\theta(B)=\theta(C)+\gamma \delta I$, and consequently, $\gamma \delta I=A-\theta(C) \in \mathcal{F}(H)$. This implies that $\gamma=0$, which further yields $B \in \mathcal{F}(H)$ as desired.

So, $\theta_{\mid \mathcal{F}(H)}: \mathcal{F}(H) \rightarrow \mathcal{F}(H)$ is a surjective Jordan homomorphism. Clearly, $\mathcal{F}(H)$ is a prime ring, that is, $A \mathcal{F}(H) B=0$ implies either $A=0$ or $B=0$. It follows that $\theta_{\mid \mathcal{F}(H)}$ is a homomorphism or an antihomomorphism [Her, Theorem 3.1]. Since $\mathcal{F}(H)$ has no nontrivial ideals, $\theta_{\mid \mathcal{F}(H)}$ is in fact an isomorphism or an anti-isomorphism. Using [Che, Theorem 3.1], we conclude that the restriction of $\theta$ to $\mathcal{F}(H)$ has one of the forms

$$
\theta(T)=S T S^{-1}, \quad T \in \mathcal{F}(H),
$$


or

$$
\theta(T)=S T^{t} S^{-1}, \quad T \in \mathcal{F}(H),
$$

for some invertible $S \in \mathcal{B}(H)$. Here, $T^{t}$ denotes the transpose relative to some fixed orthonormal basis. With no loss of generality we can assume that $S=I$.

Let us first consider the case that $\theta(T)=T$ for every bounded finite rank operator $T$. Take any idempotent $P \in \mathcal{B}(H)$ such that both kernel and image of $P$ are infinite-dimensional. By Lemma 3.4 we have $\theta(P)=\alpha Q+\beta I$ with $Q$ being an idempotent with infinite-dimensional image and kernel. Hence, $\theta((1 / \alpha) P-(\beta /(\alpha \delta)) I)=Q$. Denote $H_{1}=\operatorname{Im} P$ and $H_{2}=\operatorname{Ker} P$. Then, of course, $H=H_{1} \oplus H_{2}$ (note that in general this is not an orthogonal sum). Assume that there exists $x \in H_{1}$ such that $Q x$ and $x$ are linearly independent. Take any nonzero continuous linear functional $g$ on $H$ such that $g\left(H_{1}\right)=0$. Then $P+\lambda N$ is an idempotent for every $\lambda \in \mathbb{C}$, where $N=x \otimes g$, i.e., $N y=g(y) x$ for every $y \in H$. Hence, by Lemma 3.4 we have for every complex $\lambda$

$$
Q+\lambda N=\theta\left(\frac{1}{\alpha}(P+\alpha \lambda N)-\frac{\beta}{\alpha \delta} I\right)=k(\lambda) R_{\lambda}+m(\lambda) I .
$$

Here, $k(\lambda) \neq 0$ and $R_{\lambda}$ is an idempotent with infinite-dimensional image and kernel. As $Q+\lambda N$ is not invertible we have $m(\lambda)=0$ or $m(\lambda)=-k(\lambda)$ which yields $k(\lambda) R_{\lambda}+m(\lambda) I=-k(\lambda)\left(I-R_{\lambda}\right)$. So, with no loss of generality we have that

$$
\frac{1}{k(\lambda)}(Q+\lambda N)
$$

is an idempotent for every complex $\lambda$. This yields

$$
\left(\frac{1}{k(\lambda)}-1\right) Q=\lambda\left(N-\frac{1}{k(\lambda)}(Q N+N Q)\right) \in \mathcal{F}(H) .
$$

Therefore, $k(\lambda) \equiv 1$, and consequently, $N=Q N+N Q$. Hence, $Q($ Range $N) \subseteq$ Range $N$, and since the range of $N$ is the one-dimensional subspace spanned by $x$, this contradicts the fact that $Q x$ and $x$ are linearly independent.

So, $H_{1}$ is invariant under $Q$ and $Q_{\mid H_{1}}=\tau I_{H_{1}}$. Similarly, $H_{2}$ is invariant under $Q$ and $Q_{\mid H_{2}}=\sigma I_{H_{2}}$. With respect to the direct sum decomposition $H=H_{1} \oplus H_{2}$ we have

$$
P=\left[\begin{array}{ll}
I & 0 \\
0 & 0
\end{array}\right]
$$

and

with $\tau_{1} \neq \sigma_{1}$.

$$
\theta(P)=\alpha Q+\beta I=\left[\begin{array}{cc}
\tau_{1} & 0 \\
0 & \sigma_{1}
\end{array}\right]
$$

Take any idempotent $R$ of rank one with a matrix representation

$$
R=\left[\begin{array}{cc}
R_{1} & 0 \\
0 & 0
\end{array}\right]
$$

Clearly, $P-R$ is an idempotent, while

$$
\theta(P-R)=\left[\begin{array}{cc}
\tau_{1}-R_{1} & 0 \\
0 & \sigma_{1}
\end{array}\right]
$$

is not a sum of a scalar multiple of an idempotent and a scalar multiple of the identity unless $\tau_{1}=\sigma_{1}+1$. So, $\theta(P)=P+\sigma_{1} I$. Hence, $\theta$ maps every idempotent 
into the sum of itself and a scalar multiple of the identity. As every operator in $\mathcal{B}(H)$ can be written as a sum of five idempotents $[\mathrm{PeT}]$ we conclude that for every $T \in \mathcal{B}(H)$ we have $\theta(T)=T+\beta I$ for some $\beta \in \mathbb{C}$. Obviously, $\beta$ depends linearly on $T$. This completes the proof in our first case.

Assume now that $\theta(T)=T^{t}$ for every bounded finite rank operator $T$. Here, $T^{t}$ denotes the transpose with respect to some fixed orthonormal basis, say $\left\{e_{n}: n=\right.$ $1,2, \ldots\}$. The mapping $\psi: \mathcal{B}(H) \rightarrow \mathcal{B}(H)$ defined by $\psi(T)=\theta(T)^{t}$ is a bijective linear map satisfying:

(*) $\psi(T)=T$ for every $T \in \mathcal{F}(H)$,

$(* *) \psi(I)=\delta I$, and

$(* * *)$ if $P \in \mathcal{B}(H)$ is an idempotent with $\operatorname{dim} \operatorname{Im} P=\operatorname{dim} \operatorname{Ker} P=\infty$, then $\psi(P)=$ $\alpha Q+\beta I$ for some $\alpha, \beta \in \mathbb{C}, \alpha \neq 0$, where $Q$ is an idempotent with $\operatorname{dim} \operatorname{Im} Q=$ $\operatorname{dim} \operatorname{Ker} Q=\infty$.

In exactly the same way as above we prove that

$$
\psi(T)=T+f(T) I, \quad T \in \mathcal{B}(H),
$$

for some linear functional $f$, or equivalently,

$$
\theta(T)=T^{t}+f(T) I \quad \text { for every } T \in \mathcal{B}(H) .
$$

Here, $T^{t}$ denotes the transpose with respect to some fixed orthonormal basis, say $\left\{e_{n}: n=1,2, \ldots\right\}$. With no loss of generality we can assume that $f=0$. We will show that this case cannot occur. Namely, let $T \in \mathcal{B}(H)$ be defined by $T e_{n}=e_{n+1}$, $n=1,2, \ldots$. Then, clearly, $T^{t} e_{1}=0$ and $T^{t} e_{n}=e_{n-1}$ for every integer $n>1$. The linear span of $e_{1}$ is a minimal nonzero element of lat $T^{t}$. It follows that lat $T$ has at least one nonzero minimal element, say $K$. Let $x$ be any nonzero vector from $K$. Obviously, the linear span of $T x, T^{2} x, T^{3} x, \ldots$ belongs to lat $T$. Moreover, it is a proper subspace of $K$ which is a minimal nonzero element of lat $T$. This contradiction completes the proof.

\section{REFERENCES}

[BPW] P. Botta, S. Pierce, and W. Watkins, Linear transformations that preserve the nilpotent matrices, Pacific J. Math. 104 (1983), 39-46. MR 84c:15014

[BrS] M. Brešar and P. Šemrl, On local automorphisms and mappings that preserve idempotents, Studia Math. 113 (1995), 101-108. MR 96i:47058

[Che] P.R. Chernoff, Representations, automorphisms and derivations of some operator algebras, J. Funct. Anal. 12 (1973), 275-289. MR 50:2934

[GLR] I. Gohberg, P. Lancaster, and L. Rodman, Invariant subspaces of matrices with applications, Wiley-Interscience, New York, 1986. MR 88a:15001

[GR] I. Gohberg and L. Rodman, On distance between lattices of invariant subspaces of matrices, Linear Algebra Appl. 76 (1986), 85-120. MR 87h:47014

[Her] I.N. Herstein, Topics in ring theory, University of Chicago Press, Chicago, 1969. MR 42:6018

[JaS $\quad$ A.A. Jafarian and A.R. Sourour, Linear maps that preserve the commutant, double commutant or the lattice of invariant subspaces, Linear and Multilinear Algebra 38 (1994), 117-129. MR 95j: 47043

[O] V. Olshevsky, A condition for the closeness of the sets of invariant subspaces of close matrices in terms of their Jordan structure, Siberian J. of Math. 30 (1989), 102-110. MR 91b:47010

[PeT] C. Pearcy and D. Topping, Sums of small number of idempotents, Michigan Math. J. 14 (1967), 453-465. MR 36:2006 
[RaR] H. Radjavi and P. Rosenthal, Invariant subspaces, Springer-Verlag, Berlin, 1973. MR 51:3924

[Sem] P. Šemrl, Characterization of matrices having rank k, Linear Algebra and Appl. 42 (1997), 233-238. CMP 98:06

Department of Mathematics, University of New Haven, West Haven, Connecticut 06516

E-mail address: jafarian@charger.newhaven.edu

Department of Mathematics, College of William and Mary, Williamsburg, Virginia $23187-8795$

E-mail address: 1 xrodm@math.wm.edu

Faculty of Mechanical Engineering, University of Maribor, Smetanova 17, 2000 MARIBOR, SLOVENIA

E-mail address: Peter.Semrl@uni-mb.si 